\title{
Philonsorbonne
}

15 | 2021

Année 2020-2021

\section{Les Lumières et la défense de la liberté du travail}

Jordan MESSERLÉ

\section{(2) OpenEdition}

Journals

Édition électronique

URL : https://journals.openedition.org/philonsorbonne/1720

DOI : 10.4000/philonsorbonne. 1720

ISSN : 2270-7336

Éditeur

Publications de la Sorbonne

Édition imprimée

Date de publication : 1 janvier 2021

Pagination : $51-70$

ISSN : 1255-183X

\section{Référence électronique}

Jordan MESSERLÉ, "Les Lumières et la défense de la liberté du travail », Philonsorbonne [En ligne], 15 | 2021, mis en ligne le 03 février 2021, consulté le 21 septembre 2021. URL : http://

journals.openedition.org/philonsorbonne/1720 ; DOI : https://doi.org/10.4000/philonsorbonne.1720

(c) Tous droits réservés 


\title{
Les Lumières et la défense de la liberté du travail
}

\author{
Jordan MESSERLÉ
}

Saint-Marc Girardin, inquiet d'un socialisme qu'il jugeait trop vivace, proposait en août 1848 une histoire aussi limpide que partisane de l'idée de travail ${ }^{1}$. Dans cette histoire, qui n'avait d'ambition que d'exorciser l'appel à « organiser» le travail dont février et le sang de juin s'étaient faits l'écho, se succédaient trois époques caractérisées par un mot d'ordre spécifique. La première trouvait sa source dans la doctrine chrétienne et ne jurait que par l'obligation du travail; la seconde, nourrie des réflexions économiques $\mathrm{du} \mathrm{XVIII}^{\mathrm{e}}$ siècle, entendait protéger le « droit du travail»; enfin, la troisième se présentait comme le triste résultat des doctrines des «organisateurs chimériques et désastreux $»^{2}$ de cette première moitié du XIX ${ }^{\mathrm{e}}$ siècle. À la succession chronologique des époques se superposait ainsi la lutte entre les principes d'appréhension sociale du travail qui définissaient chacune d'entre elles. Et, pour Saint-Marc Girardin, il est peu dire que l'histoire ne s'achemine pas nécessairement vers le meilleur. Si l'auteur loue la doctrine chrétienne pour sa condamnation de l'oisiveté, ce n'est néanmoins que le XVIII ${ }^{\mathrm{e}}$ siècle qui éluciderait les véritables droits qui s'attachent au travail. En effet, on aurait alors cherché à assurer à chacun sa liberté de travailler, c'est-à-dire à le garantir contre les privilèges corporatifs et les règlements abusifs avalisés par l'État. Le XVIII ${ }^{\mathrm{e}}$ siècle consacrerait l'Âge d'or d'une pensée de l'indépendance de l'individu qui, par le travail, peut se faire l'artisan de son propre bonheur ou, du moins, porte seul la responsabilité de

1. Saint-Marc Girardin, «Histoire de l'idée du travail», Revue des deux mondes, Vol. 23, $\mathrm{N}^{\circ} 4,15$ août 1848 , p. 553-565.

2. Ibid. 
son sort. Sitôt formulée, cette doctrine d'indépendance aurait néanmoins subi l'assaut «d'utopistes » qui, en appelant à de nouveaux règlements et à repenser le droit de propriété, signifiaient leur incompréhension de l'idée de liberté.

Cette lecture fantasmée de l'évolution des idées au XVIII ${ }^{\mathrm{e}}$ siècle est commune dans les courants libéraux de la première moitié du siècle suivant. Elle le devient d'autant plus que la multiplication des projets de réformes socialistes supposait une réaction qui, toucherait-elle à la caricature, se jouait aussi sur le terrain spéculatif. L'invocation des mânes des glorieux ancêtres du siècle précédent devient ainsi un exercice pour ressusciter les "vrais » principes de l'organisation sociale, par trop obscurcis par la témérité de certains réformateurs. Cette relecture conduit donc les partisans d'une régulation du marché du travail laissée à la seule concurrence à considérer que la deuxième moitié du XVIII ${ }^{\mathrm{e}}$ siècle a été, seule, capable de donner une conception légitime de la liberté du travail. Lecture qui suppose non seulement d'ignorer certaines voix discordantes, mais surtout de simplifier des débats théoriques qui s'inscrivent dans un contexte historique et des cadres argumentatifs précis.

Sans doute les philosophes et les précurseurs de la pensée économique ${ }^{3}$ s'accordent-ils en partie dans leur critique des communautés de métier. En effet, ce rejet suit habituellement un schéma qui recoupe le refus plus général des privilèges, même si tous ne lui donnent pas une égale importance ${ }^{4}$. En ce sens, la critique des corporations, aux côtés de la défense de la liberté du commerce ${ }^{5}$, semble bien, comme l'a souligné Steven Kaplan, un laboratoire

3. Que l'on pense, en France, aux disciples de Gournay et de la «science du commerce » ou de Quesnay et de la physiocratie. Rappelons que l'économie ne constituait pas alors un champ du savoir autonome.

4. Turgot, qui aime à se dire à la fois disciple de Gournay et de Quesnay, reproche ainsi aux physiocrates dans sa correspondance avec Dupont de Nemours de ne pas insister suffisamment sur les entraves à la liberté du travail que sont les communautés de métier et le compagnonnage (Lettre du 20 février 1766, in Gustave Schelle, Euvres de Turgot et documents le concernant, Paris, Felix Alcan, 1913-1923, T. II, p. 504-515).

5. Un problème rarement souligné par les commentateurs tient à la nature du lien qui unit défense de la liberté du travail et défense de la liberté du commerce. L'ambiguïté dérive du problème de l'extension à donner à la notion de commerce au XVIII et du contexte historique dans lequel se joue essentiellement la défense de la liberté du commerce (celle du commercé de blés, crucial dans une économie traversée par des crises de sous-production). En droit, la liberté du commerce inclut la liberté du travail. Forbonnais dans l'article " Commerce» de l'Encyclopédie (Encyclopédie ou Dictionnaire raisonnée des sciences, des arts et des métiers, Paris, Briasson, Durand David Le Breton, 1751-1765, Vol III, 1753, p. 690b-699b, qu'il reprend par ailleurs en 1754 dans le chapitre I de ses Éléments du commerce) le définit de façon générale comme «une communication réciproque» qui «s'applique tout particulièrement à la communication que les hommes font entre eux des productions de leurs terres et de leur industrie ». Le chapitre II des Éléments du commerce (« De la concurrence ») fait encore de la concurrence entre travailleurs au sein de l'État « la base principale de la liberté du commerce ». De même, et malgré ce qui les oppose, Lemercier de la Rivière dans L'Ordre naturel et essentiel des sociétés politiques (1767) assimile le travail à un produit qui s'échange sur un marché (voir en particulier les chap. XXXVII et XXXVIII) et relève donc du commerce. Néanmoins, Forbonnais précise dans son article pour l'Encyclopédie que les 
pour la formation d'un « libéralisme des Lumières » ${ }^{6}$. Mais l'expression a le défaut de suggérer qu'il aurait existé une doctrine politique unanimement partagée dont le rejet des corporations serait la manifestation. Or derrière l'homogénéité de critiques qui dénoncent les effets pervers des structures d'Ancien Régime, tous ne s'accordent pas sur un même modèle de relations économiques de substitution, ni - et il s'agit du grand thème de cette deuxième moitié de siècle - sur la façon dont la garantie de la liberté du travail participera à concilier les intérêts divergents que compte la société. En effet, il n'était pas dit qu'à la suppression des corps intermédiaires ne succèdent de nouvelles formes de dominations; et donc que l'éventuelle utopie d'une régulation du travail laissée au marché ne présente des fondements fragiles. Autrement dit, si défendre la liberté du travail signifiait substituer aux corporations un ordre concurrentiel où l'individu constitue la seule unité pertinente, alors il fallait montrer que chacun avait effectivement intérêt à une telle substitution.

Cette étude entend ainsi revenir sur les défenses d'un travail libéré de l'emprise des corps intermédiaires qui se font jour dans la deuxième moitié du XVIII ${ }^{\mathrm{e}}$ siècle $^{7}$. En cela, elle s'attache à des arguments qui anticipent la naissance d'un marché du travail concurrentiel et formeront une partie de la grammaire libérale du siècle suivant. Toutefois, non seulement il n'y a pas

principaux acteurs du commerce sont avant tout (a) les «détailleurs » qui achètent des produits de la terres ou de l'industrie pour les revendre dans la limite des frontières de l'État, (b) les «manufacturiers » qui organisent le travail de ceux qu'ils emploient, (c) les «négociants » qui échangent les productions nationales avec l'étranger. Symétriquement, Lemercier de la Rivière jouera sur l'extension de la notion de commerce selon qu'il entend condamner le "trafic », c'est-à-dire la spéculation sur la valeur des marchandises, ou se référer avant tout au commerce des biens de subsistance lors de la querelle des blés. En ce sens la relation d'implication entre liberté du commerce et liberté du travail (syntagme d'ailleurs rare dans les textes) se détermine contextuellement, et la liberté du travail concerne avant tout le marché de l'offre de travail de ceux qui n'ont «que leurs bras » pour vivre (c'est-à-dire les travailleurs agricoles sans propriété, les ouvriers et les artisans).

6. Steven Kaplan, La Fin des corporations, Paris, Fayard, 2001. Voir en particulier le chapitre I. Kaplan définit ce libéralisme des Lumières comme «le point de vue économique, social et politique qui fait de la liberté le fondement de la condition et de l'existence individuelle » (p. 8).

7. Précisons immédiatement qu'une perspective philosophique ou d'histoire des idées qui, division du travail universitaire oblige, prend essentiellement appui sur des textes, ne doit pas être aveugle à leur historicité. Par exemple, il faut bien entendu admettre que les corporations ne renvoient pas à une réalité unifiée au XVIII ${ }^{\mathrm{e}}$ (la construction de cette unité relevant ellemême d'une stratégie propre à leur critique). Turgot, qui se rêve leur fossoyeur en 1776, admet parfaitement que le travail est déjà libéré de leurs règles dans bien des villes et quartiers (voir les notes qu'il adjoint à sa première œuvre publiée, la traduction des Questions importantes sur le commerce à l'occasion des oppositions au dernier bill de naturalisation des protestants étrangers, op. cit., T. I, notamment la note de la page 462). À ce sujet, outre l'ouvrage précité de S. Kaplan, on peut consulter son article « Idéologie, conflits et pratiques politiques dans les corporations parisiennes du XVIII ${ }^{\mathrm{e}}$ siècle », Revue d'histoire moderne et contemporaine, 2002, $\mathrm{N}^{\circ}$ 49-1, p. 5-55. Ou encore, parmi bien d'autres références historiques possibles, l'article d'Alain Thillay sur l'organisation du travail dans le Faubourg SaintAntoine («Le faubourg Saint-Antoine et la liberté du travail sous l'Ancien Régime», Histoire, Économie et société, vol. 11, № 2, 1992, p. 217-236). 
de marche irrémédiable de l'idée à la chose, mais ces arguments eux-mêmes sont le foyer de tensions que les contemporains percevaient clairement. En particulier, le passage d'une théorie des droits de l'individu - qui prétend fonder la liberté du travail sur le droit de propriété - à une théorie de la conciliation optimale des intérêts, suppose des conditions qui sont loin d'être accessoires pour la cohérence du discours et suscitent de vifs débats. En effet, quel sens y a-t-il à fonder la liberté du travail sur la liberté individuelle d'appropriation si une grande partie des travailleurs est condamnée à être privée de propriété ? En quoi les travailleurs les plus pauvres ont-ils vraiment à gagner à cette substitution? Si l'on accepte que l'emphase sur la liberté individuelle contre l'arbitraire des corporations révèle un « libéralisme des Lumières », celui-ci s'apparente donc moins à une doctrine constituée et consensuelle, qu'à des philosophèmes, certes récurrents, mais débattus. En cela, la critique des corporations et la défense de la liberté du travail n'accouchent pas d'un libéralisme monolithique, mais de trois éléments qui deviendront constitutifs de la pensée libérale ${ }^{8}$ : (I) une critique de l'action politique qui risque toujours de desservir l'intérêt de la société, (II) une philosophie des droits de l'individu qui s'accompagne, sous peine d'inconséquence, (III) d'une justification de l'inégalité comme composante nécessaire du meilleur ordre social possible.

Précisons que nous allons ici adopter deux principes de méthode: (1) d'abord, une exigence de systématicité en insistant sur les points de convergence dans les défenses de la liberté du travail plutôt que sur les divergences théoriques. Ainsi, pour mettre au jour des modèles d'argumentation, nous serons conduits à rapprocher des auteurs qui peuvent s'opposer sur d'autres points (les membres du «réseau» Gournay ne partagent pas les perspectives de la «secte» physiocratique, pour ne rien dire de la position hybride de Turgot; tout comme les philosophes qui prennent part au débat ne se prononcent pas nécessairement unilatéralement en faveur d'une théorie économique constituée). (2) Ensuite, nous considérons que des enjeux de philosophie politique essentiels ne

\footnotetext{
8. Si le cadre de cet article ne permet pas de longues considérations de méthode, précisons que nous ne succombons pas à une téléologie qui considéreraient les arguments étudiés à partir des relectures du siècle suivant. On peut, à ce titre, défendre deux hypothèses de lecture qui ne semblent pas contradictoires avec toute rigueur méthodologique : (1) La critique des corporations suscite des arguments qui seront, consciemment (et constamment), repris au $\mathrm{XIX}^{\mathrm{e}}$ siècle chez des écrivains politiques qui se plaisent à en faire un moment politique fondateur de la lutte contre l'organisation du travail. (2) En ce sens, il y a bien une filiation intellectuelle, mais il est évident que les arguments que l'on retrouve au XVIII ${ }^{\mathrm{e}}$ siècle se forment dans un cadre institutionnel irréductible aux réinterprétations postérieures. De plus, s'il y a un "libéralisme des Lumières" qui informe le libéralisme du siècle suivant (rappelons, néanmoins, qu'un auteur du XVIII ${ }^{\mathrm{e}}$ siècle aurait peiné à comprendre le sens politique que nous conférons à l'adjectif « libéral »), cet héritage mis en scène au XIX ${ }^{\mathrm{e}}$ siècle à des fins stratégiques (sinon idéologiques) ne doit pas faire oublier (a) que tous les philosophes du XVIII ${ }^{\mathrm{e}}$ siècle ne sont, bien entendu, pas « libéraux» (pensons, par exemple, à l'importance de la référence républicaine chez Rousseau ou Mably), et (b) que les philosophèmes « libéraux » identifiables sont étayés par des architectures théoriques diverses selon l'auteur qui les mobilise.
} 
s'expriment pas uniquement dans les textes des auteurs consacrés par l'histoire de la philosophie. En cela, la réappropriation, sinon le travestissement, de certains schèmes philosophiques (notamment rousseauistes) chez des théoriciens ou pamphlétaires que l'on ne considère pas habituellement comme "philosophes", nous semble légitime pour comprendre les caractéristiques essentielles des théories politiques qui s'imposent lors du «tournant économique ${ }^{9}$ » de cette deuxième moitié du XVIII ${ }^{\mathrm{e}}$ siècle.

\section{Une généalogie critique}

\section{1) La sédimentation historique de l'arbitraire}

Les passes d'armes contre les corporations connaissent un moment retentissant lorsque Simon Clicquot-Blervache publie, en 1758, un ouvrage dirigé contre les maîtrises et l'inspection des manufactures sous le titre Mémoire sur les corps de métiers ${ }^{10}$. Le texte examine méthodiquement les griefs que l'on peut adresser à une organisation corporative du monde du travail : (1) le nombre d'apprentis que chaque maître est autorisé à former est bien trop faible et induit une situation de monopole défavorable au consommateur, (2) la longueur de l'apprentissage et du compagnonnage semble ridiculement longue au regard de la maîtrise des savoirs et techniques qui sont exigés dans chaque profession, (3) le statut inégalitaire des corporations favorise arbitrairement les fils de maitre lors de l'admission à la maitrise (ils sont, notamment, exemptés du «chef d'œuvre »), (4) la réception à la maîtrise présente un coût qui en exclut, de fait, la majorité des ouvriers, seraient-ils talentueux, au profit de ceux qui n'ont que le bénéfice de l'argent.

Avant même de parler des effets économiques des communautés de métiers, ces dernières sont coupables d'instaurer au sein du peuple des privilèges qui favorisent la naissance et la richesse au détriment des qualités individuelles requises dans l'exercice de la profession ${ }^{11}$. Loin de remplir leur prétendu rôle de garantes de la qualité de la production, elles seraient le siège d'une distinction sociale fondée sur la naissance, en un sens

9. Steven Kaplan, Raisonner sur les blés. Essais sur les Lumières économiques, Paris, Fayard, 2017, introduction.

10. Simon Clicquot-Blervache, Mémoire sur les corps de métiers qui a remporté le prix au jugement de l'Académie d'Amiens en l'année 1757, La Haye, 1758. Le texte est aussi édité la même année sous un titre différent: Considérations sur les compagnies, sociétés et maîtrises, Londres, 1758. Gournay a participé à la rédaction du texte.

11. D’Holbach synthétise ainsi le propos : «Les privilèges exclusifs, les jurandes, les droits exigés par les communautés, etc., sont des obstacles opposés à l'industrie qui empêchent l'indigent d'améliorer son sort. S'il faut être riche pour avoir le droit de travailler, quelle ressource restera-t-il aux pauvres pour subsister? » (Éthocratie ou le gouvernement fondé sur la morale, in Euvres Philosophiques, Tome III. Paris, Alive édition, 2001, p. 653). 
homologue à celle qui sépare les roturiers des grands. L'article « Maîtrises » de l'Encyclopédie, qui en propose une critique en règle, ne manque pas de le souligner :

Mais c'était trop peu que les riches et les grands eussent envahi les fonds, les terres, les maisons; il fallait encore établir les maîtrises, il fallait interdire aux faibles, aux indéfendus l'usage si naturel de leur industrie et de leurs bras ${ }^{12}$.

Les critiques n'hésitent d'ailleurs pas à présenter le compagnonnage comme une «espèce de servitude $»^{13}$, qui permet aux membres dominants $\mathrm{du}$ corps de jouir plus longtemps du travail des aspirants sans être contraints de le rémunérer à son juste prix. Les corporations induiraient ainsi un renversement des valeurs : ce qui devrait recevoir des louanges se voit étouffé (l'habileté), alors que l'institution récompense ce qui devrait susciter l'opprobre (l'incompétence mâtinée d'aisance financière ou d'une naissance heureuse) ${ }^{14}$. Toutefois, si la nocivité sociale des corporations est telle, on peut à bon droit s'étonner de leur longévité. Comment est-on parvenu à cette situation désastreuse qui semble nuire à tous, excepté quelques privilégiés ? A fortiori, comment cette situation a-t-elle pu se maintenir ?

Or, au-delà du détail de la critique de tel ou tel règlement corporatif, les voix qui s'élèvent s'unissent souvent dans le choix d'une même méthode pour dénoncer la sédimentation de l'arbitraire corporatif: le retour à leur origine pour mieux en dénoncer le vice ${ }^{15}$. Diderot, dans les premières pages de la Lettre sur le commerce de la librairie (rédigée en juin 1767 mais qui ne sera pas publiée de son vivant), affirme ainsi considérer l'abolissement «entier et absolu» des corporations comme «un pas de plus vers un

12. Joachim Faiguet de Villeneuve, article « Maîtrises », Encyclopédie, éd. cit., Vol IX, 1765. De façon générale les Encyclopédistes ne dissimulent pas leur opposition aux corporations et à leurs statuts. Les articles qui les concernent prennent souvent la forme d'un réquisitoire pour leur transformation, sinon leur abolition (voir aussi Diderot, article « Chef D'œuvre », vol III, 1753 ; Forbonnais, article « Communauté », vol III, 1753 ou encore Jaucourt, article « Lettre de maîtrise », Vol IX, 1765). Diderot insiste tout particulièrement sur l'injustice et l'arbitraire de corps politiques qui n'admettent en leur sein que ceux qui ont eu la grâce de naître riches ou fils de maître. Les plus souvent les articles de l'Encyclopédie consacrés à une communauté de métier en retranscrivent aussi les règlements.

13. Clicquot-Blervache, op. cit., p. 15.

14. « [...] ainsi, l'ouvrier habile, mais pauvre, ne peut sortir de l'esclavage et de l'indigence ; et l'ouvrier ignorant, mais assez aisé pour acheter une maîtrise, peut s'enrichir. La fortune n'est plus la récompense des talents, vice politique, absolument contraire à l'émulation », ibid., p. 22.

15. Exceptons de ces critiques le pamphlet retentissant de l'abbé Coyer rédigé sous la forme de fiction (Gabriel-François Coyer, Chinki, histoire cochinchinoise qui peut servir à d'autres pays, 1768). Ouvrage de circonstance qui venait appuyer les tentatives de réformes des corps de métier du contrôleur général des finances L'Averdy (qui entendait faciliter l'accès à la maîtrise). La fiction permet de tourner en ridicule l'arbitraire des règlements corporatifs en proposant au lecteur de suivre les déboires d'un propriétaire d'une Cochinchine imaginaire qui, prenant acte du peu de considération du gouvernement pour la culture des terres, tente de placer ses enfants comme apprentis. 
gouvernement plus sage $»^{16}$. Le chemin de cette suppression doit être tracé par l'enquête historique: l'élucidation des causes de l'origine se fait le tribunal de la légitimité ou de l'illégitimité de l'institution, puisque «c'est dans l'historique des lois et de tout autre règlement qu'il faut chercher les vrais motifs de suivre ou de quitter la ligne tracée $[\ldots] \gg{ }^{17}$.

Ce retour critique à l'origine peut néanmoins suivre deux modèles qui, sans être contradictoires, ne se retrouvent pas également chez tous ceux qui entreprennent une histoire critique des corporations. On peut en effet montrer (a) soit que les corporations n'ont jamais répondu à aucune utilité sociale, et ne doivent donc leur existence qu'au triomphe politique momentané d'un intérêt particulier qui se sédimente institutionnellement ; (b) soit que la structure corporative répondait à un besoin passé, mais que ce dernier est aujourd'hui frappé d'inactualité. Le corollaire de cette deuxième option étant que l'institution n'en cesse pas moins de produire des effets qui, de bons qu'ils étaient, deviennent nuisibles. Or ce sont précisément ces décalages que le sage politique doit éviter, sous peine d'infliger à la société le fardeau du maintien à l'existence de ce qui ne servait qu'à résoudre un problème circonstancié.

\section{2) La loi, l'histoire et le péril de l'institution}

La critique des corporations donne donc lieu à un exercice qui devient topique, dans la deuxième moitié du XVIII ${ }^{\mathrm{e}}$, pour qui entend justifier une réforme institutionnelle : remonter à l'origine afin de déterminer si les causes qui ont prévalu à la naissance de l'institution agissent toujours ou non. L'évaluation de la transformation ou de l'inertie de ces causes fournit alors un critère pour décider de la légitimité de la réforme. Cette " généalogie » historique des causes qui, contrairement à Rousseau, prétend tout sauf commencer par écarter tous les faits, devient typique d'une critique libérale qui refuse que la tradition ou l'habitude soient les seuls critères de légitimité de la loi ou d'une institution. Certes, la méthode ne permet pas de définir à elle seule les critères de la bonne institution; mais elle fonde, du moins, la critique de celles qu'elle juge frappées d'anachronisme.

Gournay ne s'y trompe pas dans le Mémoire sur les communautés de métiers qu'il adresse à la chambre de commerce de Lyon en Février 1753. Une critique efficace des corporations suppose de revenir aux premiers moments de leur institution en France, et de dévoiler le jeu de quelques fabricants qui ont abusé de l'ignorance économique du gouvernement pour faire passer leur intérêt particulier pour l'intérêt de tous ${ }^{18}$. Que l'on considère une origine plus vertueuse des corporations, en prétendant qu'elles ont permis dans un premier temps de stimuler l'amour du travail et

16. Denis Diderot, Lettre sur le commerce de la librairie, Paris, Hachette, 1861, p. 4.

17. Ibid., p. 5.

18. Vincent de Gournay, «Mémoire sur les communautés de métiers adressé à la Chambre de commerce de Lyon », in Mémoires et lettres, Paris, Institut Coppet, 2017. 
l'apprentissage de techniques propres à chaque métier $^{19}$, ne change rien à l'affaire : "la loi doit changer, puisque les circonstances ont changé ${ }^{20}$. Le terme de "circonstance » revient sans cesse dans les débats politiques de cette deuxième moitié de siècle : sorte de reviviscence de la fortune machiavélienne avec laquelle l'habile politique doit composer, tout en prenant garde que les succès d'hier peuvent toujours se heurter aux changements des causes qui en expliquaient jusqu'alors la réussite.

Cette rhapsodie de la circonstance permet de soulever les limites de la connaissance humaine en insistant sur la part d'indétermination qui s'attache à toute action politique : il est impossible de prévoir sur le temps long les conséquences d'une réforme. Les circonstances, à un moment ou un autre, viennent condamner tout appétit réglementaire trop rigide, et vouent aux gémonies l'hubris politique qui entendrait préserver des mouvements de l'histoire des institutions nécessairement condamnées à l'inadéquation par rapport au motif de leur création.

Turgot développe le thème dans l'article «Fondation» de l'Encyclopédie ${ }^{21}$, dont l'objectif déclaré est de «dégoûter des fondations nouvelles et détruire un respect superstitieux pour les anciennes!». Quelle que soit notre foi dans les lumières de celui qui prétend ériger une institution politique durable (une fondation), le nombre de causes à prendre en considération sera toujours trop grand pour qu'il puisse assurer l'utilité intemporelle de son projet. Quelle que soit la pureté de l'intention, il se produit fatalement une distorsion entre la fonction que l'institution est censée remplir et le rôle qu'elle remplit effectivement :

Je veux bien supposer qu'une fondation ait eu dans son origine une utilité incontestable; qu'on ait pris des précautions suffisantes pour empêcher que la paresse et les négligences ne la fassent dégénérer, que la nature des fonds la mette à l'abri des révolution du temps sur les richesses publiques: l'immutabilité que les fondateurs ont cherché à lui donner est encore un inconvénient considérable, parce que le temps amène de nouvelles révolutions qui font disparaitre l'utilité dont elle pouvait être dans son origine, et qui peuvent même la rendre nuisible.

L'édit de 1776, qui supprime pendant un court moment les corporations, reprendra une argumentation analogue: ces corps intermédiaires sont assimilées à des institutions qui ne se perpétuent que par inertie, par leur capacité à se présenter comme naturelles au corps social ${ }^{22}$. Or puisque ces institutions qui semblent causa sui, ne relèvent en réalité que de la

19. Clicquot-Blervache, op. cit., Première partie, p. 25.

20. Ibid., p. 25.

21. Encyclopédie, éd. cit., Vol. VII, 1757, p. 72b-75b. Notons que Robert Castel propose de voir ce texte comme "l'essence de la philosophie politique du libéralisme » (Les Métamorphoses de la question sociale: une chronique du salariat, Paris, Gallimard, 1999, p. 282).

22. «Enfin, l'habitude prévalut de regarder ces entraves mises à l'industrie comme un droit commun » (« Édit de suppression de février $1776 »$, in op. cit., T. V, p. 242). 
sédimentation contingente de l'intérêt de quelques-uns; il devient nécessaire d'affirmer la liberté du travail à la fois contre leur arbitraire et l'adoubement juridique de l'intérêt particulier d'un corps.

Le passage d'un diagnostic historique critique à la promotion positive de la liberté individuelle du travail ne va néanmoins pas de soi (pourquoi ne pas aménager la structure des corporations ?). Comprendre les tentatives pour fonder la liberté du travail, en particulier le travail juridique de Turgot, suppose d'être attentif à deux arguments typiques des transformations du discours philosophique dans les années 1760-1770: d'une part la liberté $\mathrm{du}$ travail découle d'un droit naturel qui prévaut sur toute institution contingente faite par les hommes; d'autre part, seule la suppression des corporations entendues comme corps intermédiaires permet de préserver l'intérêt du public.

\section{Le fondement par le droit naturel : une tentative vouée à l'échec?}

\section{1) Du droit de propriété à la liberté du travail}

La dénonciation de corporations frappées d'un vice originel ou du sceau de l'inactualité n'implique pas, en effet, de leur substituer un marché du travail entièrement libre où la régulation de l'emploi individuel serait laissé au seul mécanisme de l'offre et de la demande. La critique pouvait aussi conduire à des réformes pour atténuer leur structure oligarchique (ce qui, de fait, a été plus ou moins le programme adopté jusqu'à leur abolition définitive en 1791). L'appel à la suppression des corporations supposait, quant à elle, de montrer que l'individu jouit de droits inaliénables et radicalement inconciliables avec ces formations historiques contingentes. En ce sens, le monde corporatif pouvait être conjuré par une philosophie des droits fondamentaux de l'homme en société. Les écrits des «économistes » autoproclamés qui gravitent autour de Quesnay et de son héritage sont ainsi la matrice d'une critique du monde corporatif qui, en réactualisant la pensée mythique de l'origine des sociétés, détermine à quelles conditions l'ordre social peut être légitime ${ }^{23}$. Bien sûr, ils éviteront de mobiliser le mot « contrat ${ }^{24}$ » qui laisserait supposer que la société est un tout artificiel dont la

23. Sur l'originalité des physiocrates dans l'histoire de la philosophie du XVIII ${ }^{\mathrm{e}}$ siècle et de leur théorie des droits de l'individu, voir notamment Dan Edelstein, On the Spirit of Rights, Chicago and London, The University of Chicago Press, 2018.

24. Lemercier de la Rivière n'emploie que celui de «réunion». Voir en particulier la prosopopée qui illustre les clauses de l'association politique dans le chapitre conclusif de L'Ordre naturel et essentiel des sociétés politiques où le terme revient à plusieurs reprises (Londres, Jean Nourse, 1767, Chap. XLIV, p. 443-444). Un peu plus loin, Lemercier précise : « nous cherchons à consolider le droit de propriété, et point du tout à l'énerver : nos vues et nos intérêts communs sont de garantir la jouissance de ce droit dans toute la plénitude, dans toute l'étendue qu'il avait avant de songer à nous réunir en société particulière; or, avant cette réunion, il était de l'essence du droit de propriété, que nous fussions tous également libres d'en retirer la plus grande somme possible de jouissances [...]» (je souligne, ibid., p. 244). 
formation suppose l'aliénation de certains droits dont l'individu jouissait dans l'état de nature (en cela, si l'on excepte Rousseau, ils sont plutôt dans l'air du temps où l'on se méfie des fictions contractualistes). Il importe en effet aux physiocrates d'insister sur la continuité entre état de nature et société politique afin de montrer que ces droits naturels, certes, se transforment peut-être, mais qu'à aucun moment l'individu ne les perd.

Lemercier de la Rivière propose ainsi, dans L'Ordre naturel et essentiel des sociétés politiques (1767), d'envisager le droit naturel de pourvoir à sa conservation comme le premier des droits naturels de l'individu dont vont logiquement découler les suivants ${ }^{25}$. En effet :

[...] il est évident que le droit de pourvoir à sa conservation renferme le droit d'acquérir, par ses recherches et ses travaux, les choses utiles à son existence, et celui de les conserver après les avoir acquises ${ }^{26}$.

Pour Lemercier, le droit de se conserver suppose donc celui d'acquérir, car ce premier droit implique la possibilité de l'appropriation par le travail pour s'assurer des moyens de cette conservation. Quelle que soit la validité de l'inférence, elle permet à Lemercier de distinguer entre trois types de propriétés qu'il considère logiquement liés: (1) le droit de pourvoir à sa conservation suppose que l'homme dispose de la propriété exclusive de sa personne (propriété personnelle), c'est-à-dire que les autres ne peuvent légitimement l'empêcher de jouir de son droit de se conserver ; $(2)$ ce qui implique encore la propriété exclusive des choses sans laquelle ce droit à la conservation serait nul (propriété mobiliaire); (3) Enfin, la propriété foncière est une conséquence de la nécessité de la culture des terres pour la survie de la société (qui se forme naturellement avec l'accroissement de la population). Notons que si le travail est l'opérateur de l'appropriation mobiliaire, il ne suffit pas pour justifier la propriété foncière : sa justification passe par ses effets, en tant qu'elle induit des conséquences positives pour l'ensemble de la société. Notons encore que l'ordre chronologique d'apparition de ces propriétés est inverse à leur hiérarchie dans le système économique physiocratique, et donc que le titre juridique l'emporte finalement en importance sur les «droits»du travail ${ }^{27}$. Reste que l'abbé Baudeau n'en affirme pas moins dans un ouvrage qui entendait diffuser de façon concise la doctrine physiocratique que « [...] l'exercice du Droit naturel des hommes s'étend, ou se resserre par le travail, qui remplit le premier devoir de la loi de la nature $»^{28}$.

\section{Ibid., I, chap. II.}

26. Ibid., p. 12.

27. Sur ce point, voir Philippe Steiner, «Le projet physiocratique : théorie de la propriété et lien social », Revue économique, volume 38, n 6, 1987, p. 1111-1128.

28. Nicolas Baudeau, Exposition de la loi naturelle, Amsterdam, Lacombe, 1767, p. 6. 
Se dessine donc, à partir d'une théorie qui se fonde sur une lecture partielle de John Locke ${ }^{29}$ (mais c'est celle que retiendra le libéralisme du $\mathrm{XIX}^{\mathrm{e}}$ siècle), la justification de la liberté du travail à partir du droit qu'à chacun d'œuvrer à sa jouissance par la propriété. Le travail devient l'opérateur qui me donne droit à ma part de richesses produites par la société. Bien sûr, tous les hommes ne peuvent avoir la même prétention à l'appropriation par le travail, même s'ils doivent tous être libres de mettre leurs efforts au service de leur jouissance. La différence des forces, mais aussi pour Lemercier les « circonstances» ou le « tourbillon de hasards ${ }^{30} »$, introduisent nécessairement des distinctions entre les hommes, qui n'en demeurent pas moins légitimes. Elles sont le produit de leur inégale industrie ou l'héritage de celle de leurs ancêtres. Formellement, les hommes privés de propriétés foncières jouissent d'ailleurs du même droit que tout citoyen :

Quoique moi, agent de la classe industrieuse, ne sois propriétaire que de ma personne, de mon industrie, de ma main d'œuvre, il est de l'essence de mon droit de propriété qu'il me soit permis d'en retirer la plus grande somme possible de jouissances : je dois donc être pleinement libre d'échanger mes travaux contre la plus grande somme possible de production $[\ldots]^{31}$.

Toute l'ambiguïté du propos repose sur le sens à accorder à la scansion de cet adjectif "possible» qui vient, de fait, limiter ce que peut espérer l'individu de l'usage de son droit naturel à l'appropriation par le travail. Certes, il y a continuité du droit naturel dans la société civile ; certes, comme l'a brillamment exposé Catherine Larrère, le propre des physiocrates est de passer d'une théorie du droit naturel compris comme minimum de la conservation de l'existence à une théorie du maximum de la jouissance ${ }^{32}$. Toutefois, la théorie de la formation optimale de la richesse et la division économique de la société implique que cette jouissance ne soit pas également distribuée. L'ordre économique détermine l'usage que nous pouvons librement faire de notre travail en vue de l'appropriation des produits. Autrement dit, la théorie du droit naturel que mobilisent les physiocrates $^{33}$ leur permet de justifier (au-delà des critiques opposables à

29. Lecture qui oublie les limites à l'appropriation qu'introduit dans un premier temps le rapport à autrui (injonction à ne pas gaspiller) et la modification de la théorie qu'induit l'introduction de la monnaie. Plus généralement, les physiocrates ignorent les problèmes spécifiques que pose la justification de l'appropriation chez Locke (sur le sujet, voir JeanFabien Spitz, "Locke et l'appropriation privée. À quelles conditions le droit d'exclure peut-il être justifié ? », Raisons politiques, $\mathrm{N}^{\circ} 73,2019$, p. 39-59).

30. Op. cit., p. 16.

31. Ibid., p. 452 (je souligne).

32. Catherine Larrère, L'Invention de l'économie au XVIII' siècle, Paris, PUF, 1992.

33. Mais, en la matière, ils ne sont guère les seuls et l'on retrouve des réflexions similaires chez d'Holbach qui reste, parfois explicitement, proches des économistes (voir le premier discours de la Politique Naturelle ou Discours sur les vrais principes du Gouvernement par un ancien Magistrat, 1773 ; pour une prise de position claire de D'Holbach en faveur des physiocrates, on peut consulter le chapitre VIII de son Éthocratie où le gouvernement fondé sur la morale (1776) dans lequel, précise-t-il, « on ne peut rien ajouter aux vues utiles que le bien public leur a dictées », in op. cit., Tome III, p. 651). 
cette déduction) que l'individu peut légitimement attendre des jouissances de la société par son travail personnel ; en revanche, la quantité et la qualité des jouissances auxquelles il peut prétendre dépendent de sa place dans l'ordre social (et du plus ou moins grand éloignement de ce dernier de l'ordre naturel sur lequel nous allons revenir). La liberté du travail, qui fait de l'acteur économique individuel le seul élément pertinent, ne découle donc pas simplement d'un droit naturel a priori, mais suppose de considérer que cette liberté, dont la conséquence est la concurrence des travailleurs entre eux, participe de l'état dans lequel chacun reçoit le maximum variable de jouissances auxquelles il peut prétendre (en un sens, la liberté du travail constitue une condition d'un optimum au sens parétien du terme).

\section{2) Une difficile actualisation des droits}

La liberté du travail est donc en défaut de fondement si on ne cherche à la déduire que du droit individuel à l'appropriation. En effet, l'actualité de cette liberté est toujours contrebalancée par un ordre social qui détermine la nature de la propriété dont l'individu peut effectivement jouir par son travail. Certes, Baudeau peut avancer que le droit d'acquérir se définit en proportion de la quantité et la qualité du travail individuel. Mais ce que retire effectivement le travailleur ne dépend pas uniquement de lui, et dérive de sa relation aux autres classes (au sens physiocratique). Or en considérant une situation limite, rien ne dit que mon droit naturel aux moyens de ma conservation par le travail soit effectivement conservé. En effet, le degré de jouissance auquel me donne droit mon travail n'est pas défini par un rapport simple entre quantité de travail et propriété, mais implique (au moins) le rapport que j'entretiens avec les autres travailleurs d'une part, et avec la demande de travail d'autre part. En ce sens la théorie de la formation des prix, et en particulier de la formation des salaires, laisse un espoir très limité au simple ouvrier de jouir effectivement de son droit de propriété autrement que pour acquérir des biens de subsistance. Lemercier, ici dans le cas particulier des ouvriers agricoles, l'admet facilement :

Examinez bien quel est l'état de ceux dont la profession est de servir aux différents travaux que la culture occasionne; en général, vous ne verrez en eux que des hommes réduits aux consommations qu'on peut regarder comme l'étroit nécessaire ; il s'en faut bien qu'ils soient salariés en raison de l'utilité qui résulte de leurs travaux: leurs diverses professions sont communément d'une pratique si facile, qu'elles sont à la portée d'une multitude d'hommes, et d'hommes nés sans aucune sorte de richesses; par cette raison, la grande concurrence de ces ouvriers qui se forment promptement et sans frais, tient nécessairement leurs salaires au plus bas prix possible, je veux dire à un prix au-dessous duquel on ne trouve que l'indigence et la misère, fléaux toujours destructifs des classes d'hommes dont ils forment l'état habituel ${ }^{34}$.

34. Lemercier de la Rivière, éd. cit., III, chap. XXXII, p. 279. 
La raison en est simple et implacable, «nécessaire » comme ne cesse de le répéter Lemercier : d'une part, les employeurs ont intérêt à rémunérer le moins possible le travail; d'autre part, pour les travaux simples, la concurrence que se livrent le grand nombre de travailleurs potentiels conduit le prix du travail à baisser. Le salaire des ouvriers agricoles ou d'industrie, des «simples manouvriers", coïncide donc nécessairement avec le prix des productions qu'ils consomment, et «c'est sur ce prix courant que la concurrence règle leur salaire, parce que les salaires sont le gage et le signe de la part qu'ils doivent prendre dans la production $»^{35}$. Pour le dire en langage physiocratique, au sein d'un marché du travail concurrentiel, ceux qui n'ont que leurs bras pour travailler et ne disposent pas de connaissances spécifiques, voient la valeur vénale de leur travail (le prix du marché) coïncider avec sa valeur fondamentale (que Lemercier nomme aussi «prix nécessaire »), c'est-à-dire la somme des coûts de production au sens large (matière première s'il y en $\mathrm{a}$, biens de subsistance nécessaire au

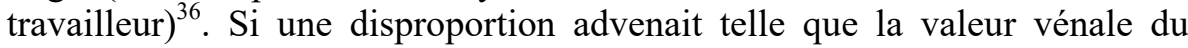
travail soit inférieure à sa valeur fondamentale ; eh bien, soit la faim ferait son ouvrage pour rétablir de justes proportions en diminuant la concurrence, soit, plus probable pour les économistes, l'ouvrier choisirait la solution rationnelle de l'émigration jusqu'au nouvel équilibre. Équilibre qui finira bien par advenir, même si «cet effet ne sera pas subit, et qu'il y a dans toute machine compliquée des frottements qui ralentissent les effets les plus infailliblement démontrés par la théorie ${ }^{37}$.

On le voit, la liberté du travail pour certains membres de la société ne donne donc droit qu'à une part restreinte des richesses produites. Lemercier se heurte d'ailleurs en 1770 à l'opposition de Galiani qui considère que le système physiocratique, en plaidant pour la libéralisation du commerce des grains, implique la négation du droit à l'existence de chacun ${ }^{38}$. Objection qui conduit Lemercier, dans sa réponse, à reformuler sa théorie du droit légitime qu'a chacun aux jouissances produites en prétendant placer le

35. Ibid., p. 282. Les physiocrates considèrent néanmoins que le salaire doit permettre à la fois l'entretien de l'ouvrier et de sa famille. On ne voit pas, toutefois, pourquoi le prix du travail ne se fixerait pas autour du nécessaire pour l'individu qui peut travailler à plus bas prix que celui qui possède une famille, si ce n'est que l'entrepreneur de travaux considère que les travailleurs qu'il emploie sont aussi des consommateurs. Jean-Baptiste Say avancera dans son Traité d'économie politique (1803, II, 8, § 4) qu'il est rationnel pour l'employeur que l'ouvrier gagne plus que sa simple subsistance afin qu'il puisse élever ses enfants, et donc que le nombre d'ouvriers soit toujours suffisant pour la masse de travaux disponibles. Ce surplus de salaire est pour lui la conséquence d'un homme qui se définit comme «capital accumulé » : il nécessite un certain nombre d'investissements avant de pouvoir participer à la production.

36. Voir notamment Quesnay, «Sur les travaux des artisans », in François Quesnay, Euvres économique complètes et autres textes, Éd. par Christine Théré, Loïc Charles et Jean-Claude Perrot, Tome II, Paris, INED, 2005.

37. Turgot, «Lettre à Hume du 25 mars 1767 », op. cit., Tome II, p. 664.

38. Ferdinando Galiani, Dialogues sur le commerce des bleds, Londres, 1770. Voir notamment les sixième et huitième dialogues. 
droit à l'existence au fondement de l'association sociale ${ }^{39}$. Toutefois, et cette argumentation est promise à un long avenir, ce droit ne doit pas être compris comme une créance que l'individu posséderait sur l'État, mais comme un effet de l'ordre économique optimal. En ce sens, la liberté du commerce (qui inclut ici la liberté du travail) est une «conséquence nécessaire du droit que tous les hommes ont à l'existence et aux moyens d'exister ${ }^{40}$, car elle seule peut le garantir sans que le reste de la société en souffre. Encore une fois, la philosophie du droit naturel ne suppose nullement un droit inconditionnel, mais un exercice de légitimation de l'ordre socio-économique le plus apte à l'actualiser.

\section{III- La nécessaire légitimité ex post : le meilleur ordre possible}

\section{1) L'ordre et la conciliation des droits}

Le droit à l'existence, que l'on oppose aux réquisits de la théorie de la distribution et de la formation des richesses des physiocrates, donne l'occasion à Lemercier de préciser les dispositions les plus favorables à «l'intérêt commun ». Or s'il admet un droit à l'existence, ce dernier n'implique aucune remise en cause ou limitation du droit de propriété. La propriété, et en particulier la propriété foncière, se justifie en effet comme une conséquence du droit à l'existence entendu comme droit à l'appropriation individuelle des moyens de subsistance. De plus, la propriété est jugée nécessaire dès lors que l'on donne pour fonction à la société de produire le maximum possible des bien agricoles.

Seule l'institution de la propriété privée permet d'aiguillonner l'intérêt des propriétaires à cultiver leurs terres en leur laissant un produit qu'ils pourront échanger (impôt déduit) contre d'autres jouissances (ils sont, en conséquence, la source de la demande de travail de la classe stérile en tant que dispensateurs du produit net). Contre Mably, qui dans ses Doutes proposés aux philosophes économistes sur l'ordre naturel et essentiel des sociétés politiques $(1768)^{41}$, avançait que l'institution de la propriété foncière

39. Pierre-Paul Lemercier de la Rivière, L'Intérêt général de l'État ou la liberté du commerce des blés (1770), in La liberté du commerce. Cuvres économiques 1765 et 1770, Paris, Slatkine édition, 2013.

40. Ibid., p. 57.

41. Voir notamment la «Lettre première à l'auteur des éphémérides du citoyen »: "Chaque habitant est destiné, selon ses talents, ses forces et son âge, à une fonction utile ; et l'État, propriétaire de tout, distribue aux particuliers les choses dont ils ont besoin. Voilà, je vous l'avoue, une économie politique qui me plait encore autant que si je n'avais pas lu ce que nos philosophes ont écrit sur la propriété foncière » (Gabriel Bonnot de Mably, Doutes proposés aux philosophes économistes sur l'ordre naturel et essentiel des sociétés politiques, La Haye, 1768 , p. 9). Sur la conception de la propriété de Mably et sa philosophie du droit naturel, on peut consulter Stéphanie Roza, «L'héritage paradoxal de John Locke dans Des droits et des devoirs du citoyen de Mably », in Philosophical Enquiries : revue des philosophies anglophones, $\mathrm{N}^{\circ} 2$, décembre 2013. 
relevait de la contingence historique et de la convention, Lemercier précise qu'elle est au contraire " volontaire et nécessaire ${ }^{42}$ comme l'action de boire ou de manger. Nécessaire car elle découle du droit naturel de chaque homme aux moyens d'assurer sa substance et, a fortiori, garantit le maximum de jouissance possible à laquelle chacun peut prétendre; volontaire car l'examen impartial de ses effets emporte l'adhésion. Une république de petits propriétaires supposerait d'ailleurs, selon Lemercier, de se priver des agréments des Arts et de l'industrie qui nécessitent une classe d'hommes libérés de la contrainte des travaux de la terre. Autrement dit, la division du travail moderne est une condition à la production optimale des jouissances. L'inégalité entre propriétaires fonciers et les exclus de cette propriété s'apparente donc à un moindre mal relativement au bénéfice que la société retire de la reconnaissance de la propriété. En réalité, précise Lemercier, il faut considérer que si la propriété foncière incite les propriétaires à cultiver leurs terres (et donc à augmenter la production), leurs produits appartiennent en fait à l'ensemble de la société (si le propriétaire entend maximiser sa jouissance, il doit échanger). À la statique du titre juridique, se substitue la dynamique des échanges économiques qui rendent possible à chacun de profiter de la production. Mais se pose alors le problème politique du critère qui fixe l'extension du droit individuel à profiter des jouissances produites par la société. Or, « ce droit des autres hommes, quoiqu'il résulte du droit d'exister, ne peut cependant être autre chose que le droit d'acquérir par leurs travaux, et de s'approprier une portion de ces productions $\gg{ }^{43}$.

La liberté du travail se justifie ici au nom du juste critère de distribution des biens produits par la terre. Un ordre social vertueux suppose pour chaque homme « la liberté d'employer ses facultés à ce que ses besoins exigent de lui ; qu'il est, par conséquent, seul et unique propriétaire de ses travaux et du produit de ses travaux $»^{44}$. Mais comme, d'une part, chaque homme dispose de ce droit à l'existence qui n'est qu'une liberté d'échanger son travail contre un part des biens produits par la terre et, d'autre part, que le propriétaire reste libre en vertu de son droit de propriété d'échanger le produit de la terre avec qui bon lui semble (donc sans blesser son intérêt), il reste à déterminer le principe qui doit borner les prétentions de chacun lors de l'échange. Or, il faut bien qu'intervienne le "despotisme de la concurrence ${ }^{45}$ qui vise à départager anonymement des hommes à la liberté formellement égale et à fixer l'extension de leurs droits.

Certes, cette concurrence suppose que le prix du travail, même dans des conditions optimales, soit à peine supérieure au nécessaire vital pour une

42. L'Intérêt général de l'État ou la liberté du commerce des blés (1770), chapitre IV, note de la page 70 .

43. Ibid., Chapitre IV, p. 72.

44. Ibid., p. 73.

45. L'Ordre naturel et essentiel des sociétés politiques, éd. cit., III, chap. XXXII, p. 272. 
partie non négligeable de la population ${ }^{46}$. Cette partie de la population, comme l'a vu Turgot, ne recoupe d'ailleurs pas la division en classes que proposent les physiocrates puisque les travailleurs agricoles de la classe productive et les ouvriers de la classe stérile partagent ici un destin commun $^{47}$. Mais cette position est un moindre mal, compte tenu du fonctionnement de l'ordre économique qui, pour maximiser les jouissances à l'échelle de la société, suppose une inégalité structurelle dans la part de jouissances produites à laquelle chacun peut prétendre. La liberté du travail, et la promotion de la concurrence qui l'accompagne, sont des conditions pour que les productions de la terre se vendent au «bon prix ». Celui-ci permet alors aux cultivateurs de dégager un bénéfice suffisant pour investir dans la culture, et amorce le cycle vertueux de formation des richesses mis en avant par les physiocrates. Toutefois, la défense de la liberté du travail contre la régulation corporative ne se comprend bien qu'à partir de la référence à un ordre qui garantit la meilleure distribution possible des jouissances produites tout en en justifiant l'inégalité.

\section{2) Intérêts particuliers et intérêt commun}

Outre cet aspect économique, la liberté du travail et l'ordre concurrentiel qui l'accompagne ont une conséquence sociale que n'ont cessé de mettre en avant ses défenseurs : elle consacre la hiérarchie de l'utile contre celle du privilège hérité. Le physiocrate («dissident») Boesnier de l'Orme peut ainsi écrire que « faire jouir chaque classe et chaque individu du droit qu'il a à la subsistance, en proportion de l'utilité dont il est à la société, est la perfection de l'ordre économique ${ }^{48}$. Autrement dit, libérer le travail permet aux choses et au travail des hommes de recouvrer leur véritable valeur jusqu'alors voilée par la logique du privilège et de l'arbitraire. La valeur légitime du travail s'établit en fonction de l'utilité sociale que lui confère le public, c'est-à-dire, ici, la demande ${ }^{49}$. La liberté du

46. Notons à ce sujet que l'augmentation du revenu disponible, s'il est investi dans la culture de nouvelles terres, ne conduit que momentanément à l'élévation des salaires avant que l'augmentation de la population ou l'immigration fassent de nouveau coïncider valeur fondamentale et valeur vénale. La société y gagne car les physiocrates, populationnistes, considèrent l'augmentation de la population comme une source de puissance. En revanche, il n'y a pas d'augmentation de la part de jouissances accessibles individuellement à la majorité des travailleurs.

47. Turgot, Réflexions sur la formation et la distribution des richesses, § LXI à LXV (op. cit., T. II, p. 570-572).

48. De l'esprit du gouvernement économique, Paris, 1775, p. 62.

49. Diderot présente les choses de façon lapidaire au § CXVI des Observations sur le Nakaz lorsqu'il se propose de ne dire « qu'un mot» au sujet de l'appartenance à une corporation : « c'est un privilège exclusif qui condamne celui qui sait travailler à ne rien faire, à être un voleur ou à mourir de faim. Si cet ouvrier est habile, il s'enrichira; si c'est un mauvais ouvrier, il sera pauvre. Le public est le seul vrai juge de sa capacité » (in Diderot, Euvres politiques, Éd. Paul Vernière, Paris, Classique Garnier, 2018, p. 432). Notons néanmoins, pour lever toute ambiguïté, que cette défense d'une hiérarchie de l'utile s'accompagne chez Diderot d'un rapport fluctuant à la physiocratie qui, d'abord enthousiaste, se fait 
travail conditionne alors la légitimité des inégalités économiques, au sens où la distinction sociale des individus fondée sur le bénéfice qu'ils retirent de leur travail est jugée se concilier avec l'intérêt de la société dans son ensemble ${ }^{50}$.

Néanmoins, cette reconnaissance sociale de l'utilité par l'échange ne peut jouer son rôle qu'à la condition que l'individu soit la variable pertinente dans ce système d'attribution de valeur. Les corporations doivent être proscrites car elles induisent la défense d'un intérêt de corps contre l'intérêt de la société en général. L'édit de février 1776 ne s'y trompe pas : " la source du mal est dans la faculté même accordée aux artisans d'un même métier, de s'assembler et de se réunir en un corps $»^{51}$. Pourquoi ? Parce que cette incorporation permet aux membres de la profession de ne se soucier que " de l'intérêt commun des membres de la société particulière ; intérêt qu'elles poursuivent avec une activité continue, au préjudice de ceux de la société générale $»^{52}$. Turgot participe ainsi à une dynamique de rationalisation économique de la pensée politique du XVIII ${ }^{\mathrm{e}}$ siècle par rapport à laquelle la théorie économique naissante cherche à se positionner. Rousseau et le Discours sur l'économie politique ${ }^{53}$ offrait ainsi la matrice théorique d'un rejet des corps intermédiaires en dramatisant l'opposition entre la volonté des sociétés particulières et la volonté générale qui caractérise l'ensemble de la société. En effet,

La volonté de ces sociétés particulières a toujours deux relations ; pour les membres de l'association, c'est une volonté générale ; pour la grande société, c'est une volonté particulière, qui très souvent se trouve droite au premier égard, et vicieuse au second ${ }^{54}$.

Cette reprise d'un philosophème rousseauiste ne se fait pas néanmoins sans un aménagement, voire sans un travestissement. Les défenseurs de la liberté du travail, qui envisagent l'horizon d'une régulation concurrentielle des hommes, peuvent effectivement trouver le modèle d'une critique du

particulièrement critique après le constat du résultat des premières mesures de libéralisation (rappelons que Diderot a relu puis commenté les Dialogues de Galiani). Pour une interprétation qui situe cette critique diderotienne de la physiocratie par rapport à la pensée républicaine, voir Christophe Hamel, «Diderot contre la physiocratie : une critique républicaine ? », in Revue d'histoire de la pensée économique, 8/2, 2019, p. 207-239.

50. Arnault Skornicki a souligné la portée de la pensée physiocratique dès lors qu'on la comprend contextuellement. Il n'est pas anodin dans une société de privilèges, où la politique se joue dans les intrigues de Cour, et où la rationalité économique se heurte à la logique de la gloire et de l'honneur, de faire du marché l'opérateur de la définition de la valeur sociale (L'Économiste, la cour et la patrie. L'économie politique dans la France des Lumières, Paris, CNRS édition, 2011).

51. Turgot, «Édit de suppression des jurandes », op. cit., T. V, p. 240.

52. Loc. cit.

53. Qui paraît en 1755 dans le Volume V de l'Encyclopédie puis en 1758 de façon séparée.

54. Rousseau, «Discours sur l'économie politique », in Euvres complètes, T. III, Paris, Gallimard, Bibliothèque de la Pléiade, p. 245. 
privilège par l'opposition du particulier et du général chez Rousseau ; mais le lexique change de façon significative. À la volonté générale se substitue donc la notion, loin d'être synonyme pour ceux qui l'emploient, de «public » puisque les privilèges corporatifs « sont originairement l'ouvrage de l'intérêt des particuliers, qui les ont établis contre le public ${ }^{55}$. Or qu'estce que le «public »? Rien d'autre qu'une agrégation d'individus dont les intérêts, bien que dissemblables dans le but poursuivi, sont identiques dans leur forme : chacun cherche à maximiser son utilité en termes de salaires, de coûts, et de prix de ventes. Il est rationnel que chacun veuille la fin des corporations car chacun embrasse tour à tour le rôle de consommateur et de producteur. En conséquence, ce qui est perdu sur un tableau est immédiatement regagné sur l'autre, malgré les représentations économiques pernicieuses (car unilatérales) que diffusent les corps de métiers. Les conditions institutionnelles qui prévalent à la formation de la volonté générale chez Rousseau ${ }^{56} \mathrm{~s}$ 'effacent au profit d'un intérêt du public qui n'est que la liberté laissée à chacun de poursuivre son intérêt égoïste. Cet intérêt, pour Lemercier de la Rivière, n'en demeure pas moins "commun " ${ }^{57}$ puisque l'ordre économique suppose que le résultat de l'action individuelle, dans une société libérée des entraves qui obscurcissent le jugement des acteurs économiques, serve nécessairement la société en général. Certes, les intérêts des particuliers peuvent s'opposer; mais cette opposition s'inscrit dans l'objectivité de la division de la société en classes économiques qui, sous condition d'une législation adéquate, sont unies par le même objectif d'augmentation du produit net. Les oppositions s'effacent à l'échelle macroéconomique dès lors que l'on possède une idée claire des lois de la formation de la richesse (pour les physiocrates, du moins, chacun peut former cette idée: d'où leur insistance sur la nécessité d'une instruction publique). Mais, une nouvelle fois, la garantie de la juste valeur du travail individuel, malgré une dynamique sociale d'oppositions des intérêts, suppose un troisième terme : un intérêt commun à l'accroissement du produit net auquel chacun participe en suivant librement son intérêt individuel. C'est-à-dire, finalement, le postulat d'une harmonie qui s'institue malgré l'individu et les vices de détails.

\section{Conclusion - Une grammaire libérale du XVIII ${ }^{\mathrm{e}}$ siècle}

Cette grammaire libérale qui se fait jour dans la deuxième moitié $\mathrm{du} \mathrm{XVIII}^{\mathrm{e}}$ siècle, tributaire des débuts d'une rationalisation économique de thèmes classiques de la philosophie politique, se caractérise par au moins trois arguments dont hérite le libéralisme du $\mathrm{XIX}^{\mathrm{e}}$ siècle :

55. Turgot, «Édit de suppression des jurandes », op. cit., p. 240.

56. Déjà dans le Discours sur l'économie politique, à plus forte raison dans le Contrat social, même si les définitions données de la volonté générale ne sont pas identiques.

57. L'Ordre naturel et essentiel des sociétés politiques, éd. cit., III, chap. XXXVIII. 
(1) D'abord, elle fournit une méthode : celle d'une généalogie historique qui décèle, dans la coalition des intérêts au sein d'une association, le risque de la consécration juridique de privilèges arbitraires qui s'instituent au détriment de l'ensemble de la société. Cette méthode permet donc de révéler et de dénoncer les conséquences de l'immixtion juridique de l'État sur le marché lorsqu'il entend rendre pérenne une institution. L'action de l'État devient douteuse, d'une part car elle peut être détournée par des intérêts puissants du service de l'intérêt commun ; d'autre part, car elle est toujours suspecte de produire sur la durée des effets contraires à l'intention initiale. Notons à ce sujet que Smith condamne, certes, les associations ouvrières qui prétendent augmenter artificiellement le prix de travaux, mais considère aussi que ce risque de détournement de la loi concerne surtout les maîtres. Compte tenu de leur profil sociologique qui les rapproche davantage des législateurs que le simple ouvrier, ils dissimulent plus facilement leurs intérêts sous le langage du droit et de l'évidence. L'absence de l'intervention de l'État dans la relation contractuelle qui unit le travailleur à son employeur se justifie alors, et avant tout, par le profit que pourraient en tirer les maîtres. L'État doit renoncer à réguler le marché, certes, car il risque d'enrayer une mécanique naturelle de production de la richesse, et donc d'accroissement du bien-être, mais aussi car son action s'expose à la corruption des intérêts des acteurs socio-économiques dominants ${ }^{58}$.

(2) Ensuite, elle propose une philosophie des droits naturels qui fait de la liberté du travail une conséquence du droit de propriété. Puisque les hommes se sont réunis pour assurer leur subsistance, alors la société doit garantir le moyen le plus juste de s'approprier une part des richesses produites en commun. Mais, dès le XVIII ${ }^{\mathrm{e}}$ siècle, cette relation entre appropriation et liberté du travail apparaît comme éminemment problématique: au sein de sociétés historiquement constituées, où la propriété a déjà été instituée de telle sorte que certains ne possèdent que leur bras, la possibilité de l'appropriation est nécessairement limitée par la structure de l'ordre social. Autrement dit, ce à quoi le travail donne droit n'est pas une conséquence directe d'un prétendu droit naturel, mais se définit selon la place que chacun occupe dans l'ordre économique de la formation des richesses. D'où l'échec de toute tentative pour fonder a priori la liberté $\mathrm{du}$ travail individuel sans justifier que cette liberté soit constitutive du meilleur ordre possible.

(3) Cette déduction de la liberté du travail à partir d'un schéma classique de la philosophie implique donc, pour reprendre une expression qui fut employée par Pierre Bourdieu, une "sociodicée » ${ }^{59}$. C'est-à-dire, ici, une justification à la fois de l'inégale distribution des propriétés et de l'inégal espoir que chacun entretient quant aux possibilités d'appropriation par son

58. Voir Adam Smith, Enquête sur la nature et les causes de la richesse des nations, I, chap. 8 : « des salaires du travail ».

59. Par exemple : Pierre Bourdieu, «Stratégies de reproduction et modes de domination », Actes de la recherche en sciences sociales, vol. 105, décembre 1994, p. 3-12. 
travail. Les physiocrates résolvent en effet le problème par une théorie de l'ordre naturel qui, sagement entretenu par le législateur-souverain, garantit que ces inégalités n'en permettent pas moins à chacun de retirer le maximum de jouissances auquel il peut prétendre (aussi limité soit-il dans certains cas). Le $\mathrm{XIX}^{\mathrm{e}}$ siècle, au travers d'un pamphlétaire comme Frédéric Bastiat, ne se lassera pas, même si c'est à partir de prémisses économiques distinctes, de répéter qu'un marché du travail régulé par la concurrence interindividuelle constitue le système le plus harmonieux, malgré les maux de détails. Néanmoins, cette justification se fonde chez les physiocrates sur un postulat fragile : celui de l'horizon d'une productivité indéfinie de la terre qui permet, au renouvellement de chaque cycle, d'espérer un accroissement du produit net (qui, rappelons-le, conduit à moyen terme à un accroissement de la population plutôt qu'à l'amélioration de la situation individuelle des plus pauvres). Or, au XVIII ${ }^{\mathrm{e}}$ siècle Turgot lui-même soulève une objection à ce postulat en esquissant l'idée d'un rendement décroissant des terres ${ }^{60}$. A fortiori, les théoriciens de la première moitié du XIX ${ }^{\mathrm{e}}$ siècle, que cela soit par Malthus ou Ricardo, ne pourront se prévaloir d'un tel optimisme et, pour reprendre une formule de Charles Gide et Charles Rist, ces économistes «pessimistes", «tout en affirmant comme Smith et les physiocrates l'identité des intérêts individuels avec l'intérêt général [...], ont fourni cent bonnes raisons de ne plus y croire; ils ont montré partout des antagonismes déconcertants, entre les propriétaires et les capitalistes, entre les capitalistes et les travailleurs $»{ }^{61}$. C'est notamment dans cet interstice théorique, rendu béant par les transformations économiques qui séparent le $\mathrm{XVIII}^{\mathrm{e}}$ du XIX ${ }^{\mathrm{e}}$ siècle, que croîtront des théories politiques alternatives, proposant de substituer la logique de l'association et d'une nouvelle implication de l'État au seul modèle d'une régulation de la liberté individuelle de travailler par la concurrence.

60. «La production suppose des avances; mais des avances égales dans des terres d'une inégale fécondité donnent des productions très différentes, et c'en est assez pour faire sentir que les productions ne peuvent être proportionnelles aux avances [...], et l'on ne peut jamais supposer que des avances doubles donnent un produit double » (Turgot, Sur le mémoire de Saint-Péravy, op. cit., T. II, p. 644).

61. Charles Gide et Charles Rist, Histoire des doctrines économiques depuis les physiocrates jusqu'à nos jours, Quatrième édition, Paris, Librairie de la société du recueil Sirey, 1922. p. 137. 